\title{
Investigation of nonionic diazo dye-doped polymer dispersed liquid crystal film
}

\author{
FARZANA AHMAD ${ }^{1}$, MUHAMMAD JAMIL ${ }^{1-3}$, YOUNG JAE JEON ${ }^{1, *}$, LEE JIN WOO ${ }^{1}$, \\ JAE EUN JUNG ${ }^{4}$ and JAE EUN JANG ${ }^{4}$ \\ ${ }^{1}$ Department of Chemistry, ${ }^{3}$ Department of Physics, Konkuk University, Seoul 143-701, Korea \\ ${ }^{2}$ Division of International Studies, University College, Konkuk University, Seoul 143-701, Korea \\ ${ }^{4}$ Department of Information and Communication Engineering, Daegu Gyeongbuk Institute of Science and Technology, \\ (DGIST), Daegu 711-873, Korea
}

MS received 1 December 2010; revised 25 May 2011

\begin{abstract}
Sudan black B (SBB) was used to investigate as the nonionic diazo dye-doped in polymer dispersed liquid crystal (PDLC) display, by polymerization-induced phase separation (PIPS) method. The maximum absorbance, contrast ratio, dichroic ratio and the order parameter of nonionic diazo dye in nemetic host (TL203) were investigated using UV-Vis polarized spectroscopy. The orientation of the dye molecules was controlled by electric field, which enabled the contrast ratio of the dye to be obtained by electrically switching. The change occurring on droplet morphologies and electro-optical properties of PDLC film with the change in contents of Sudan black dye and liquid crystals (LC) contents was investigated. We found an increase in LC droplet sizes with the increase of diazo dye and $L C$ contents. Moreover the addition of small amount of nonionic diazo dye reduced the threshold voltage $\left(V_{\text {th }}\right)$, increased off-state transmittance, enhanced the contrast ratio and decreased the response time of dye-doped PDLC. Additionally the change in transition temperature of LC and changes in LC droplet morphologies with the addition of dye were also observed. Such changes were observed with the images taken by polarized optical microscope (POM). The detail discussions on such behaviours were also made.
\end{abstract}

Keywords. Polymer dispersed liquid crystals (PDLC); polymer-induced phase separation (PIPS); droplet morphology; order parameter; dichroic dye; contrast ratio.

\section{Introduction}

In the recent years, studies on polymer dispersed liquid crystal (PDLC) films; that are composed of micron size liquid crystal (LC) droplets dispersed within polymer matrix (Fergason 1984; Drzaic 1995; Masutani et al 2006) were the subject of much interest industrially as well as institutionally; due to their wide applications ranging from switchable displays, light windows (Simoni 1997; Simoni and Francescangeli 2000) and holographic gratings (Sutherland et al 1994). Since, the presence of small external electric field, PDLC devices can be switched from scattered opaque to transparent state without the use of polarizer.

The director orientation of dispersed liquid crystal (LC) in the absence of external electric field varies randomly from droplet to droplet. In this case, the ordinary refractive index $\left(n_{\mathrm{o}}\right)$ and extraordinary refractive indexes $\left(n_{\mathrm{e}}\right)$ of liquid crystals were different from polymer refractive index $\left(n_{\mathrm{p}}\right)$ and this difference is responsible for the strong light scattered state of PDLC in voltage off-state. In the presence of sufficient external electric field, the LC director becomes aligned towards the direction of electric field and as a result, PDLC film appears transparent. The transmittance of light from

\footnotetext{
*Author for correspondence (yjjeon@konkuk.ac.kr)
}

PDLC film depends on the matching of ordinary refractive index of LC droplet to polymer refractive index, i.e. $n_{\mathrm{o}} \sim$ $n_{\mathrm{p}}$. The light scattering properties of PDLC films depend strongly on many other parameters that include droplet size, droplet shape, droplet number density, interfacial interactions, etc. (Han et al 2000). Moreover the off scattering and high ON transmittance were responsible for high PDLC display contrast.

The past few decades studies performed on dichroic-dyes doped with liquid crystal dispersed in polymer matrix predicted that doping of dyes into PDLC, is a useful technique to get good black state for higher contrast (Fergason 1985) and to enhance the optical response of LC device. Such dyedoped LC material dispersed into polymer matrix is known as guest-host polymer dispersed liquid crystal (GHPDLC) or dichroic dye-doped PDLC (DD-PDLC) (Fergason and Calif 1983; Montgomery and Vaz 1987; Lee et al 2002; Lin et al 2004). These DD-PDLCs have the potential to outperform conventional reflective-type twisted nematic LC display in some applications since they do not require polarizers, leading to increase in the reflectivity, viewing angle (Wu and Fuh 2005), produce higher contrast than isotropic dye and also reduce the threshold voltage.

Several research groups have studied the dye-doped PDLC films in nonalign and align configurations (West and 
Ondris-Crawford 1991; Petti et al 2002; Zhou et al 2003), using azo dye (Lee et al 2002; Fuh Andy et al 2004; Liu et al 2009) and anthraquinone dichroic dye (Kumar et al 2006; Kumar and Raina 2007; Malik et al 2008) to seek the effect of dye on morphology and electro-optical properties of PDLC films.

Meanwhile the azoaromatic compounds were extensively investigated due to their potential use in nonlinear and optical devices, for employing stable and optically induced birefringence (Rochon et al 1992) and dichroism. Among the photoresponsive dyes reported so far, azobenzene derivatives are commonly used in LC-based photonic materials. Owing to the azobenzene derivatives' reversible trans-cis isomerization by photo irradiation (Urbas et al 2004), one can manipulate not only just the optical properties, but also the optical anisotropy of the surrounding LCs as well. However, for the most of azobenzene derivatives, UV light is needed to trigger the photo switching, which usually degrades the physical properties of the LCs (Wen et al 2004).

To avoid this issue, it is favourable to choose an azo-dye whose trans-cis isomerization can be triggered by visible light (Liu et al 2009). Sudan black B appears to be a good candidate in this case, since its absorption band of trans isomer is typically in the visible range, the effect of which is negligible on the LCs. Moreover Sudan black B has high stability due to its rod like molecular structure (Ghanadzadeh and Zakerhamidi 2004). As per our knowledge diazo dyedoped PDLC films were not studied extensively. Thus the aim of this study is to explore the effect of nonionic diazo dye on morphology and electro-optical properties of dye-doped PDLC film. In this study, we varied the dye, the LC concentrations and investigated the absorbance of light by dye, its contrast ratio, dichroic ratio and order parameter in LC. In addition, the effect of dye on morphology and electrooptical properties of DD-PDLC films were also studied.

\section{Experimental}

\subsection{Materials}

The nonionic diazo-dye Sudan black B (Sigma-Aldrich) was used as solute (guest) in the eutectic nematic mixture of TL203 (nematic mixture of pentyl cyanobiphenyl blend with fluoro- and chloro-substituted mesogens) (E Merck, Germany) with positive dielectric anisotropy $(\Delta \varepsilon=11.0)$ and used as anisotropic host. The dye was dissolved in the liquid crystal with the concentrations of $0.11,0.22,0.325$, 0.418 and $1.0 \mathrm{wt} \%$; these concentrations were simply taken as $0 \cdot 1,0 \cdot 2,0 \cdot 3,0.4$ and $1.0 \mathrm{wt} \%$, respectively. The nonionic diazo dye-doped polymer dispersed nematic liquid crystal (DD-PDLC) films were prepared by polymer-induced phase separation (PIPS) method at room temperature conditions. For this purpose, the UV cureable monomer PN393 (Merck, Germany) was used. The monomer with different weight ratios (20-24 wt\%) was mixed into different weight ratio mixtures of dye and $\mathrm{LC}$ by several hours stirring. The different compositions of DD-PDLC are shown in table 1.
Table 1. Polymer, LC and dye composites for dye-doped PDLC cells.

\begin{tabular}{lccc}
\hline Sample & TL203(Wt\%) & SBB (Wt\%) & PN393 (Wt\%) \\
\hline a-1 & 80 & - & 20 \\
a-2 & 80 & $0 \cdot 1$ & 20 \\
a-3 & 80 & $0 \cdot 2$ & 20 \\
a-4 & 80 & $0 \cdot 3$ & 20 \\
a-5 & 80 & $0 \cdot 4$ & 20 \\
b-1 & 78 & - & 22 \\
b-2 & 78 & $0 \cdot 1$ & 22 \\
b-3 & 78 & $0 \cdot 2$ & 22 \\
b-4 & 78 & $0 \cdot 3$ & 22 \\
b-5 & 78 & $0 \cdot 4$ & 22 \\
c-1 & 76 & - & 24 \\
c-2 & 76 & $0 \cdot 1$ & 24 \\
c-3 & 76 & $0 \cdot 2$ & 24 \\
c-4 & 76 & $0 \cdot 3$ & 24 \\
c-5 & 76 & $0 \cdot 4$ & 24 \\
\hline
\end{tabular}

The micro bead spacer (Sekisui chemicals, Japan) with a $20 \mu \mathrm{m}$ diameter is used to control the cell thickness. In this work the material was used as is without further purification.

\subsection{Cell preparation}

Indium/tin oxide (ITO) coated glass plates were used as transparent electrodes for the measurement of the guest-host cells. The planner orientation (homogeneous alignment) of the guest and host cells was achieved by surface treatment of the glass surfaces with polyimide (PI) coating and additional rubbing process. The guest-host cells were prepared by sandwiching the solutions between glass plates $\left(2 \times 4 \mathrm{~cm}^{2}\right)$ which were filled with $10 \mu \mathrm{m}$ micro bead spacer. The DDPDLC mixtures were filled within indium tin oxide (ITO) coated glass plates by capillary action. The cell gap was fixed with $20 \mu \mathrm{m}$ diameter micro bead spacer (Sekisui Chemicals, Japan). The DD-PDLC samples were cured under UV light at $365 \mathrm{~nm}$ wavelength at room temperature.

\subsection{Measurement of maximum absorption of dye, contrast ratio, dichroic ratio and order parameter}

To determine the maximum absorption wavelength of Sudan black B dye, the absorption of UV light by dye with different concentrations in LC at voltage off was measured. To measure the contrast ratio the polarized parallel absorption spectra of the guest-host mixtures in the visible spectral region at zero and saturated applied field (40 volts) was measured using UV-Vis spectrophotometer. The contrast ratio was defined as the ratio between maximum absorption at on and off states, which was calculated as:

$$
\mathrm{CR}=A_{\text {off }} / A_{\text {on }} \text {. }
$$


In accordance to the theory (Ghanadzadeh and Zakerhamidi 2004) for positive dichroic dye, the dichroic ratio $R$ and the order parameter $S$ of the dye were calculated by the formulas $\left(A_{\|} / A_{\perp}\right)$ and $S=(R-1) /(R+2)$, respectively. The dichroic ratio $(R)$ and order parameter $(S)$ of the SBB dye dissolved in the nematic liquid crystal host was calculated from the absorption of light polarized parallel $\left(A_{\|}\right)$and perpendicular $\left(A_{\perp}\right)$ to the liquid crystal alignment. The dichroic ratio $R$ was calculated at maximum absorption wavelength.

\subsection{Methods}

The absorption of dye at different dye contents and polarized parallel absorption spectra of the guest-host mixtures in the visible spectral region at zero and saturated applied field were measured using Minolta (Japan) UV-Vis spectrophotometer (model UV-3500d). The polarized absorption spectra of the dissolved dyes were taken with the electric field on (40 saturated voltage).

The LC droplet morphology in DD-PDLC films were viewed under polarized optical microscope (Olympus Model BX-60) at a magnification of $10 \times$ fitted with charge coupling device (CCD) digital camera interfaced with a computer. The thermo-optical response was measured by polarized optical microscope (POM) equipped with hot stage. The electro-optical responses of DD-PDLC were studied when an electric field is applied to the sample.

\section{Results and discussion}

\subsection{Absorption spectra and contrast ratio}

Figure 1 shows the UV-Vis absorption spectra of Sudan black B with $0 \cdot 1,0 \cdot 2,0 \cdot 3,0.4$ and $1.0 \mathrm{wt} \%$ contents in LC. The figure 1 shows two maximum absorptions peaks $\left(\lambda_{\max }\right)$ at 420 and $600 \mathrm{~nm}$ wave length in visible range of the absorption for Sudan black B dye in TL203 LC. The largest absorption peak was absorbed at $600 \mathrm{~nm}(0.536 \%$ absorbance) wavelength. Further the dichroic ratio and order parameter were measured only at $600 \mathrm{~nm}$ wavelength. The spectra showed an increase in absorption of dye with addition of dye contents. The maximum absorption of dye was observed for $1 \mathrm{wt} \%$ guest-host cell.

Figure 2(a) illustrates the absorption spectra of SBB dye at zero and saturated applied voltage. The contrast ratio of the dye was calculated by using the formula, $C R=A_{\text {off }} / A_{\text {on }}$. Here $A_{\text {off }}$ is the absorption of light of dye in LC host at zero electric field and $A_{\text {on }}$ is the absorption of light at 40 volts (saturation voltage). With zero electric field $\left(E_{\mathrm{s}}=0\right)$, the liquid crystal molecules were in a uniform parallel orientation and the dye molecules were oriented with the long molecular axis parallel to the electric field vector of the polarized light (i.e. parallel to the nematic director). At $40 \mathrm{~V}$, the voltageabsorption curve is saturated; in fact, a minimum absorption

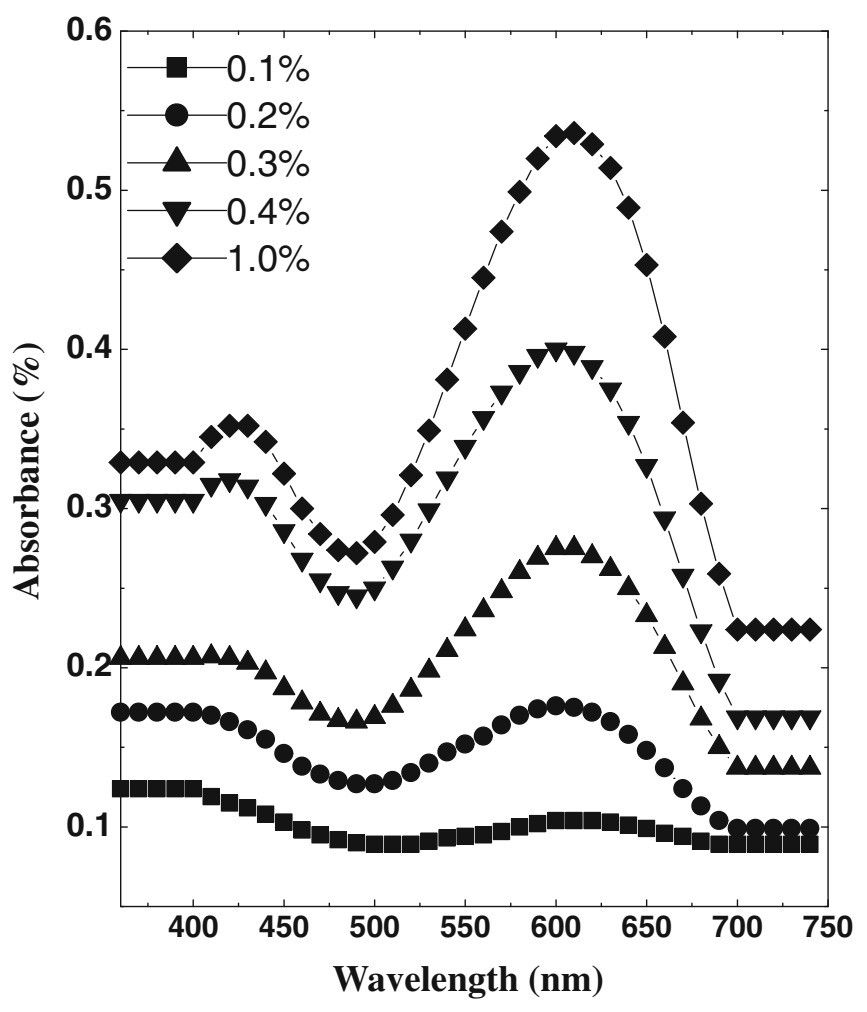

Figure 1. Absorption spectra of dye with different contents dissolved in TL203 LC host.

at $A_{\text {on }}$ of the dye was recorded. The contrast ratio obtained for SBB dye in TL203 is attained as 1:1.25.

\subsection{Dichroic ratio and order parameter}

Figure 2(b) shows the polarized absorption spectra of $1 \%$ nematic solution of Sudan black B at polarized parallel $\left(A_{\|}\right)$ and perpendicular $\left(A_{\perp}\right)$ to the liquid crystal alignment using a planar alignment cell.

The dichroic ratio $(R)=\left(A_{\|} / A_{\perp}\right)$ and order parameter $S=(R-1) /(R+2)$ of Sudan black B dye were calculated by using the absorption spectra at polarized parallel $\left(A_{\|}\right)$and perpendicular $\left(A_{\perp}\right)$ to the liquid crystal alignment. Since the direction of the transition dipole moment of the dye molecules were largely parallel to the long molecular axis, the order parameter $S$ of the dye were calculated from the equation $S=(R-1) /(R+2)$. Figure 2(b) shows the absorption at polarized parallel $\left(A_{\|}\right)$as 1.02 and at perpendicular $\left(A_{\perp}\right)$ as 0.75 to the liquid crystal alignment. The dichroic ratio observed here was 1.336. The order parameter value was also measured by using the above stated equation and its value appeared to be $0 \cdot 1$. It should be considered that the value of $S$ might differ from the order parameter for the molecular axis $S_{\mathrm{M}}$. This case is true when there is an angle between the transition moment and long molecular axis. The $C R$ directly relates to order parameter for 
the transition moment, while the molecular structure was considered to relate with order parameter for the molecular axis (Ghanadzadeh and Zakerhamidi 2004).

It can be seen that the CR value obtained in this work was slightly smaller than the dichroic ratios values obtained. It should be noted that the dichroic ratios were calculated from the absorption of light polarized parallel $\left(A_{\|}\right)$and perpendicular $\left(A_{\perp}\right)$ to the liquid crystal alignment. As $A_{\text {on }}$ (electric field direction dependence) and $A_{\perp}$ were not really the same quantities, the dichroic ratio and the contrast ratio values were also different.

Figure 2(b) indicates that $A_{\|}-A_{\perp}$ is positive for the absorption band of the dye when the transition corresponding to the visible band was polarized in the direction of the long molecular axis, and can be considered as parallel transitions (i.e. $\pi-\pi^{*}$ ). In other words, during the $\pi-\pi^{*}$
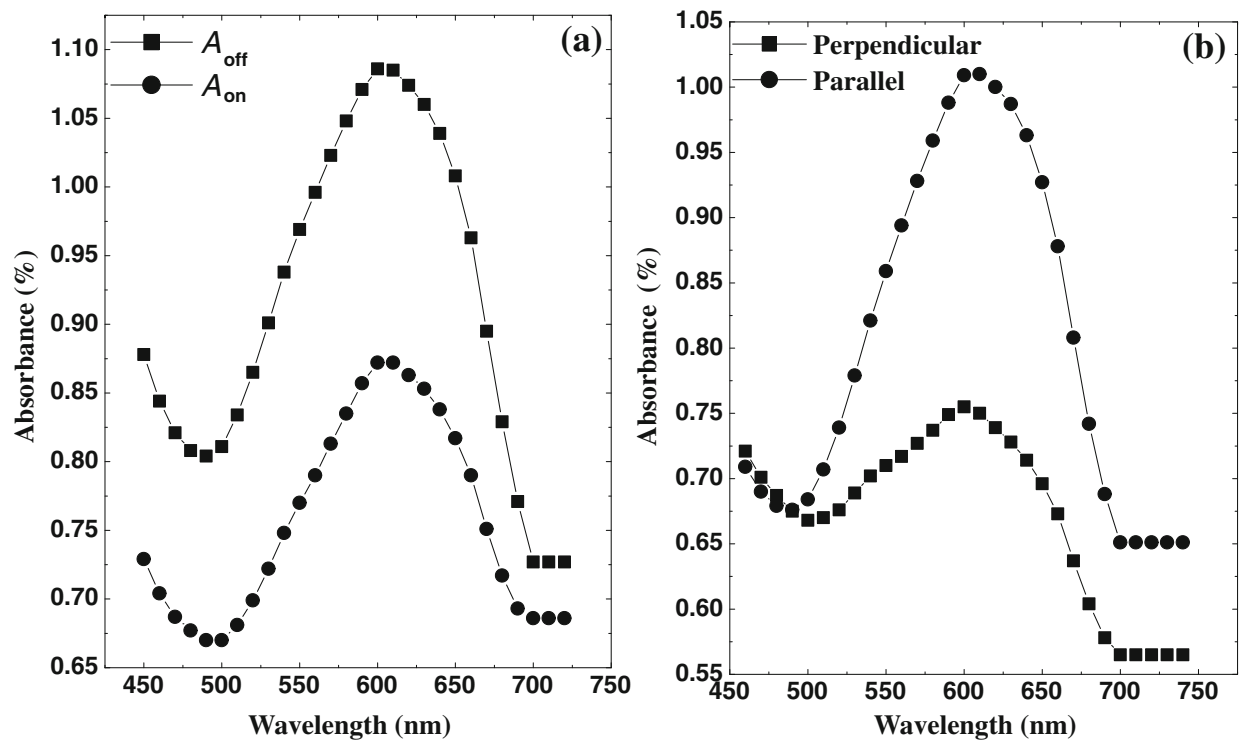

Figure 2. Absorption spectra of $1 \mathrm{wt} \%$ dye dissolved in LC (a) at zero- and saturated (40)-applied field and (b) at polarized parallel and perpendicular to the LC.

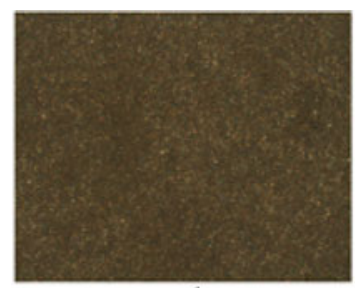

a-1

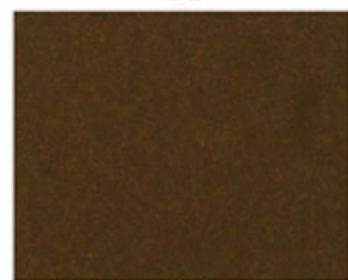

b-1

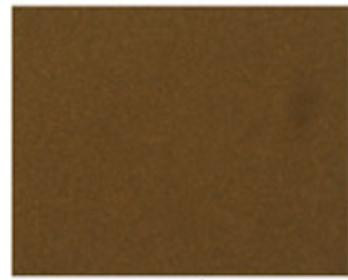

c-1

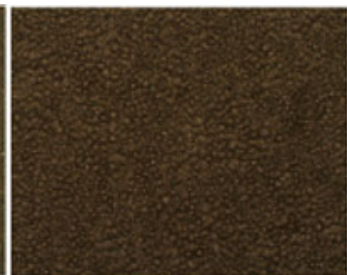

a-2

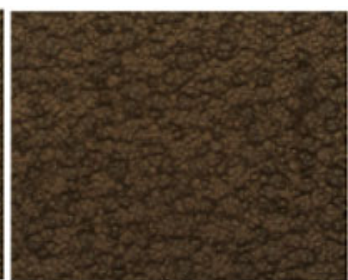

a-3

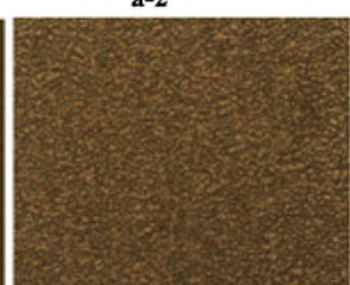

b-2

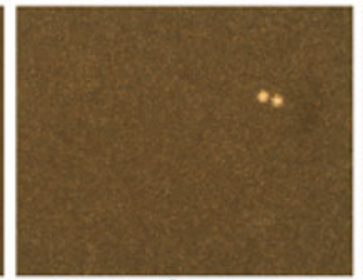

c-2

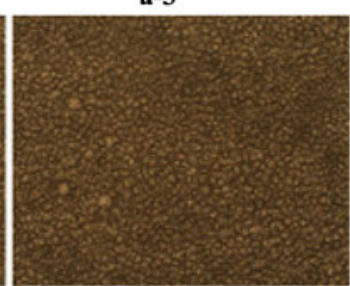

b-3

c-3

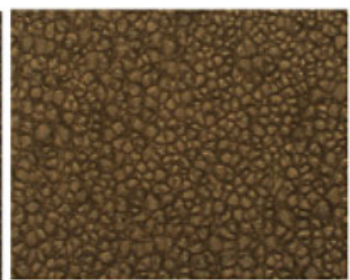

a-4

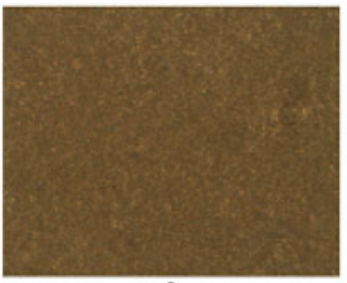

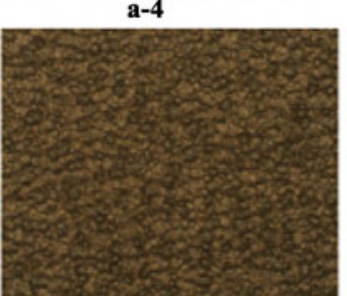

b-4

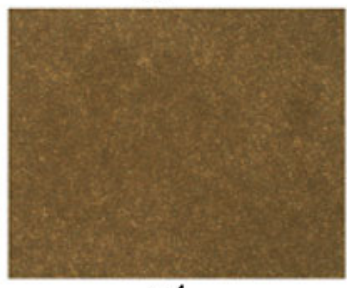

c-4

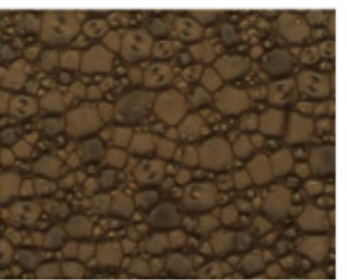

a-5

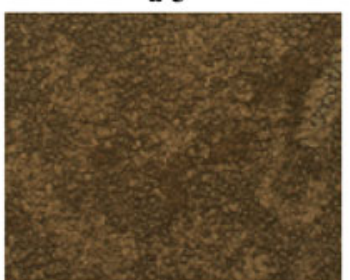

b-5

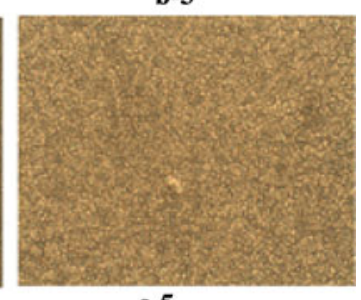

c-5

Figure 3. Micro droplet morphology of DD-PDLC at different LC concentrations: (a) 80/20 wt $\%$ (b) 78/22 wt $\%$ and (c) 76/24 wt $\%$ at varied nonionic diazo dye contents at zero volts. 
transition, a net displacement of charge occurs parallel to the molecular plane (Ghanadzadeh and Zakerhamidi 2004).

\subsection{Droplet morphology of nonionic diazo Sudan black B-doped PDLC}

Figure 3(a-c) shows observed LC droplet morphologies from optical microscope at $10 \times$ magnifying power at voltage offstate for non-aligned dye-doped PDLC (DD-PDLC) cells at varied LCs compositions, such as 80, 78 and $76 \mathrm{wt} \%$ ), respectively; whereas the concentrations of diazo dye was varied as $0.1,0.2,0.3$ and $0.4 \mathrm{wt} \%$. Figure 3 shows the increase in droplet size of DD-PDLC with the addition of dye for all the LC compositions. This can be more clearly seen for 80 and $78 \mathrm{wt} \%$ LC contents. The figure 3(a) displays smaller and more uniform distribution of LC droplets at lower dye concentration $(0.1$ and $0.2 \mathrm{wt} \%)$ than those at the higher concentration of dye $(0.3$ and $0.4 \mathrm{wt} \%)$ for $80 \mathrm{wt} \%$ LC contents. The coalescence of LC droplets also appeared at such concentrations. The better droplet uniformity helps to enhance light-scattering efficiency when the droplet size was comparable to the wavelength of scattered light (Springer and Higgins 2000). The smaller droplets formation in low dye concentration were due to the less free volume available for the dispersed low dye concentration droplets in the polymer matrix (Malik et al 2008). Further, it is clear from the UV-VIS absorbance spectra that the dye in the films absorbed UV light and this absorbance increased with the increase of dye concentration. The high absorption of UV light by dye molecules might reduced the UV curing intensity and this might resulted into incomplete polymerization of polymer which in turn produced bigger LC droplet size (Montgomery et al 1991). While for other LC weight fractions the well-defined droplet morphology was observed.

Figure $3(\mathrm{a}-\mathrm{c})$ also shows the decrease in droplet size with the decrease of LC weight fractions. The decrease in droplet size with decreasing LC fraction was observed previously (Lovinger et al 1994; Carter et al 1997; Han et al 2000) and explained by changes in the extent of polymerization at the onset of phase separation. Possibly with the high LC contents fraction, the PDLC mixture moved closer to the phase boundary resulting in a less developed polymer matrix during phase separation and this resulted into longer time for the LCs to form large domains and coalescence was seen.

As stated earlier, both droplets shape and LC configuration play important roles in governing the light-scattering properties of PDLC film. Figure 4 exhibits the combination of the nemetic liquid crystal droplet morphologies i.e. bipolar, radial, spheroidal and toroidal found simultaneously in DD-PDLC as observed by polarized microscope at $0.4 \mathrm{wt} \%$ dye concentration, comprised of $80 \mathrm{wt} \%$ LC contents. Figure 4 (b, c) displays the formation of toroidal and spheroidal or ellipsoidal LC droplets in DD-PDLC, respectively. The toroidal configuration appeared spontaneously in the PDLCs due to the collapse of polymer shell covering the LC droplets. Possibly the dye molecules absorbed at the polymer/LC interface weaken the polymer wall and tend to collapse the polymer wall. A reduction in interfacial energy can increase the number of toroidal LC droplets (Denk et al 1990; Mei and Higgins 1998; Hall and Higgins 2003). Other works have also reported the formation of toroidal configuration in pure PDLC and similar is the case for DD-PDLC (Xu et al 1996; Malik and Raina 2010).

The radial and bipolar configurations of droplets found in DD-PDLC are shown in figure 4(a). It was stated earlier (Kumar and Raina 2007) that most polymers tend to induce a parallel alignment of director field of nematic liquid crystals at interface, so that bipolar droplets are most often found in PDLC films; whereas the radial configuration also is most often found in DD-PDLC films.

Figure 5 displays the influence of external electric field on LC droplet orientation at room temperature conditions. The optical axis orientation, corresponding to the average nematic director alignment, varies randomly from droplet to droplet at zero-applied electric field. On applying the electric field across the film, we observed a bulk change of colour and orientation of the bipolar LC droplet even at very low applied (4 volts) electric field (figure 5(b)). An alteration in orientation in bipolar droplets was observed, in such a way

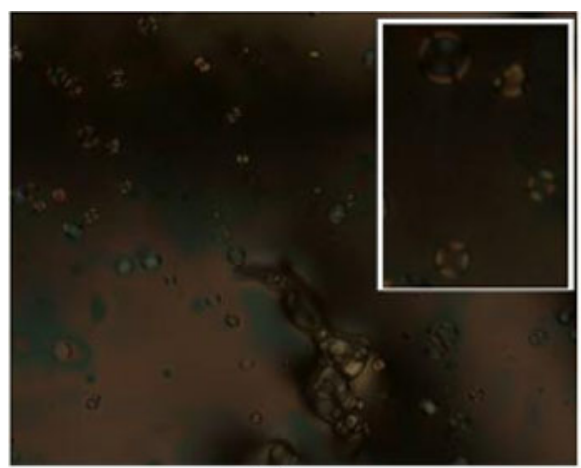

(a)

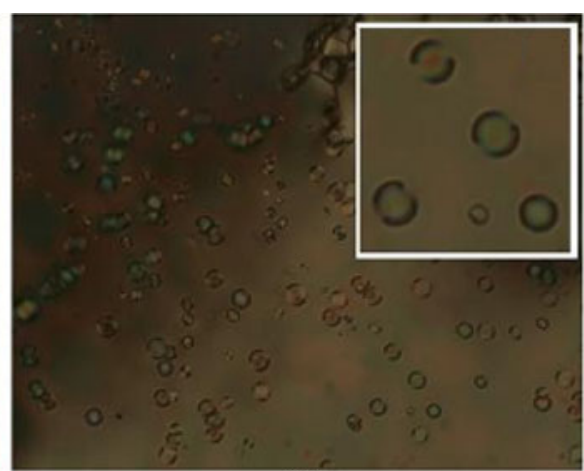

(b)

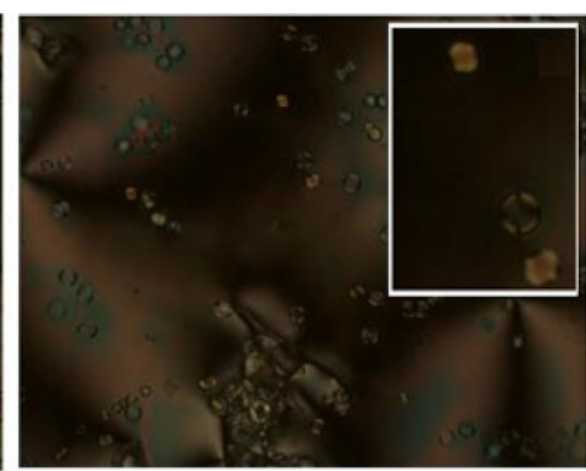

(c)

Figure 4. Several LC droplets images of (0.4 wt \%) of dye-doped PDLC with 80/20 wt \% LC composition: (a) radial and bipolar, (b) toroidal and (c) spheroidal droplet configurations. 


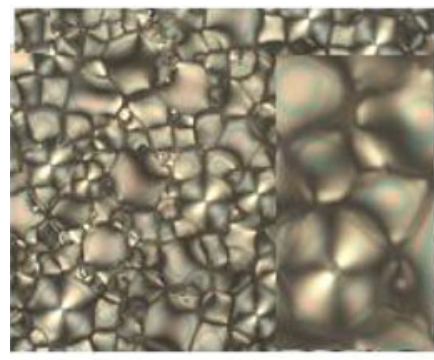

(a)

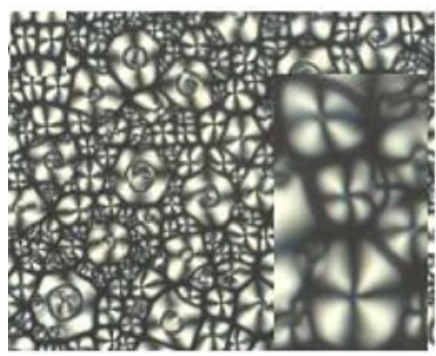

(e)

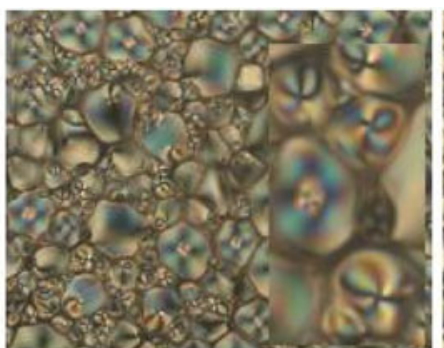

(b)

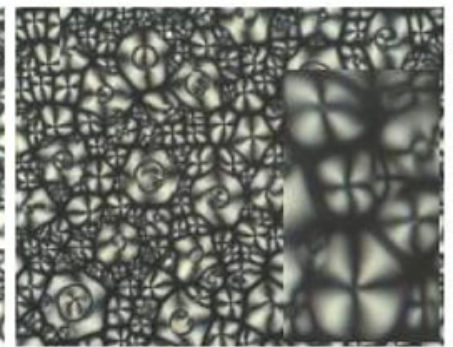

(f)

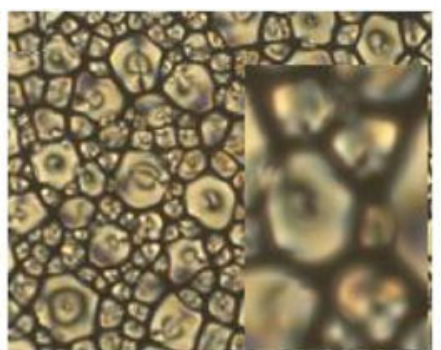

(c)

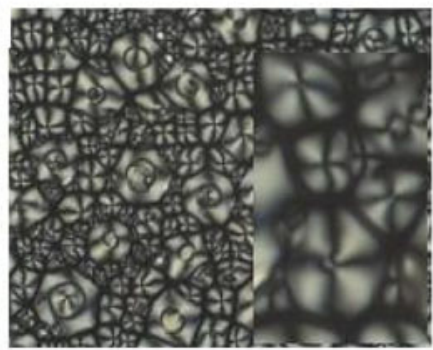

(g)

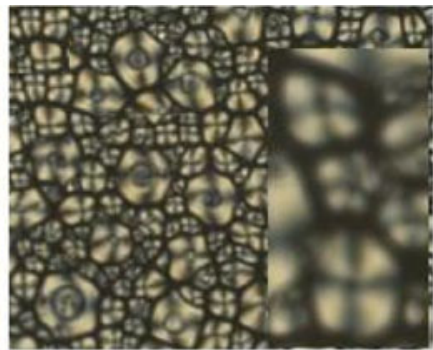

(d)

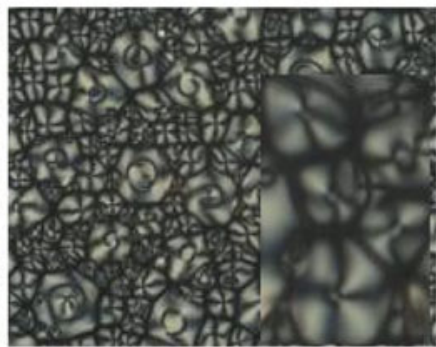

(h)

Figure 5. Droplet orientation of dichroic (0.4 wt \%) PDLC with 80/20 wt \% LC composition under electric field: (a) 0 Vp-p, (b) 4 Vp-p, (c) $5 \mathrm{Vp}-\mathrm{p}$, (d) $10 \mathrm{Vp}-\mathrm{p}$, (e) $15 \mathrm{Vp}-\mathrm{p}$, (f) $20 \mathrm{Vp}-\mathrm{p}$, (g) $30 \mathrm{Vp}-\mathrm{p}$ and (h) $50 \mathrm{Vp}-\mathrm{p}$.

that the bipolar axes tend to align along the direction of the applied field. Figure 5(d) shows that at 10 volts the small droplets appeared with a well-defined maltese type crosses and bigger droplets with twisted arrangement in the centre. The maltase type arrangement of LC droplets with voltage was reported earlier (Malik and Raina 2010). The bigger twisted orientation of droplets was considered as a fusion of 2-4 bipolar droplets to make this unstable intermediate maltase structure. This might be due to the coalescence of LC droplets which were adjusted finally to well-defined maltese-type cross after stabilization voltage.

The above stated maltase type morphology was also observed for the low dye concentrations of 0.2 and $0.3 \mathrm{wt} \%$, but at relatively low voltage (not shown here). The change in droplet orientation observed at high applied voltage for higher dye contents might be due to the change in droplet morphology, increase in conductivity of LC droplets, partial increase in viscosity of the composite film and strong anchoring with the polymer walls (Malik and Raina 2010). Whereas for other low dye contents and for low LC contents we could not observe these images as the LC droplet size was small to be observed under the POM limit.

\subsection{Phase transition temperature}

We observed the variation in phase transition nemetic to isotropic $\left(T_{\mathrm{N}-\mathrm{I}}\right)$ temperature of nemetic liquid crystal with the addition of dye by POM equipped with hot stage. For this purpose, the dye was dissolved with different weight percentage in nemetic liquid crystal. Further a drop from these solutions is sandwiched between the two ITO-coated glass plates and placed over the hot stage. The rate of increase in temperature was fixed at $0 \cdot 2^{\circ} \mathrm{C} / \mathrm{min}$. The phase transition temperature of LC cell without the dye molecules was $75^{\circ} \mathrm{C}$. A large change in $T_{\mathrm{N}-\mathrm{I}}$ was found with the addition of even small amount of dye $(0.1 \mathrm{wt} \%)$ into LC and fall in LC $T_{\mathrm{N}-\mathrm{I}}$ point from $75^{\circ} \mathrm{C}$ to $73^{\circ} \mathrm{C}$, however it was found that $T_{\mathrm{N}-\mathrm{I}}$ is further independent of dye concentrations. So further increase in dye concentration did not affect significantly on $T_{\mathrm{N}-\mathrm{I}}$ values. This temperature was found to be almost constant for all the further investigated dye concentrations. In order to understand this behaviour, it is important to consider the absorption of heat energy as well as light by dichroic dye. This might be the reason that the significant change in transition temperature was not observed.

The thermal effect on nematic LC configuration and colour for $0.4 \%$ dye contents with $80 \mathrm{wt} \% \mathrm{LC}$ contents are seen in figure 6. It was found that at $30^{\circ} \mathrm{C}$ LC (figure 6(a)) droplets in DD-PDLC exhibit predominately the disordered bipolar configuration with two point defects at the ends of the diameter along the bipolar axis. Further, the change in droplet colour and reorientation of LC droplets was observed at $62.7^{\circ} \mathrm{C}$ temperature. Finally at $67^{\circ} \mathrm{C}$, which was $6^{\circ} \mathrm{C}$ below the $T_{\mathrm{N}-\mathrm{I}}$ of the mixture, the presence of an isotropic phase become clear (dark spots); and at last, at $71^{\circ} \mathrm{C}$, the nematic-isotropic phase transition was more evident.

At elevated temperature, but below $T_{\mathrm{N}-\mathrm{I}}$ the DD-PDLC showed well-ordered bipolar droplets (with cross and two point defects on the bipolar axis) and spherodial droplets orientations. The reorientation of LC droplets with increase in temperature without voltage, may be due to the decrease in surface anchoring energy with the increase in temperature. Hence the increase in temperature enhanced the transmittance of PDLC at applied voltage as reported earlier (Malik and Raina 2010). 


\subsection{Electro-optical properties}

A voltage-transmittance (V-T) characteristic was measured for various dye concentrations at $25^{\circ} \mathrm{C}$ for DD-PDLC. Figure 7 shows the transmittance curves of dye-doped PDLC and the data calculated from this is given in table 2 . The minimum transmittance, maximum transmittance, contrast ratio, threshold voltage and driving voltage were generally chosen as $T_{0}, T_{\text {sat }}, C R, V_{\text {th }}, V_{\text {on }}$, respectively. Figure 7 and table 2 show that maximum transmittance is decreased with the addition of dye. It can be concluded that lower dye-doped PDLC showed higher transmission than higher dye-doped PDLC at voltage ON state. It was stated earlier that higher absorption on higher dye concentration leads to lower transmission of the guest-host device (Malik and Raina 2010). Thus lower dye-doped PDLC films give better transmission

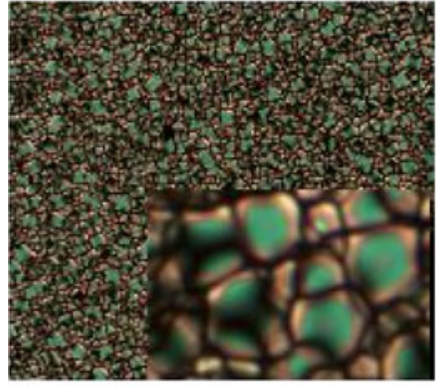

(a)

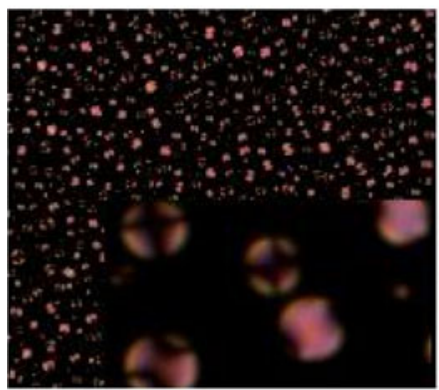

(e)

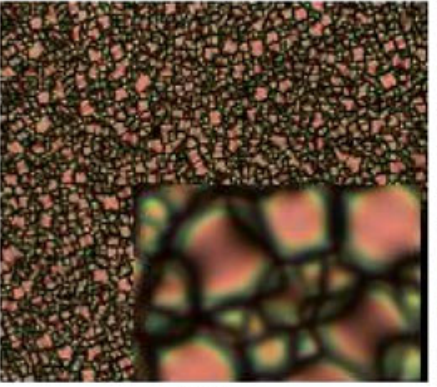

(b)

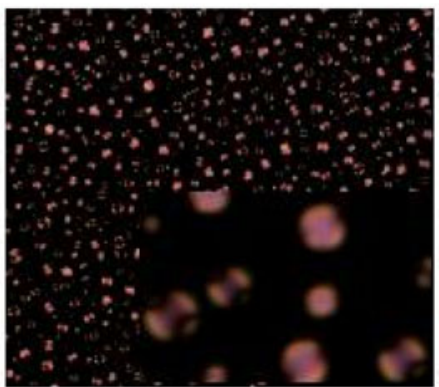

(f)

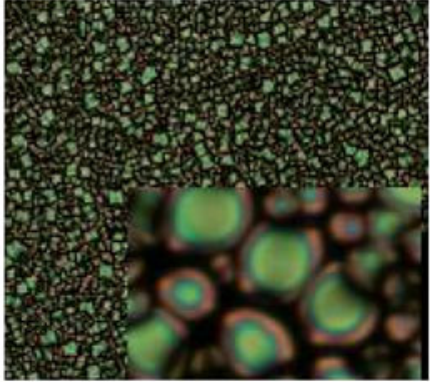

(c)

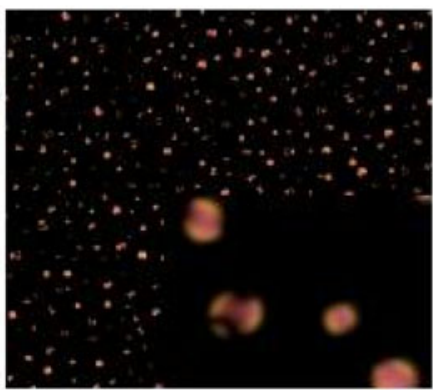

(g)

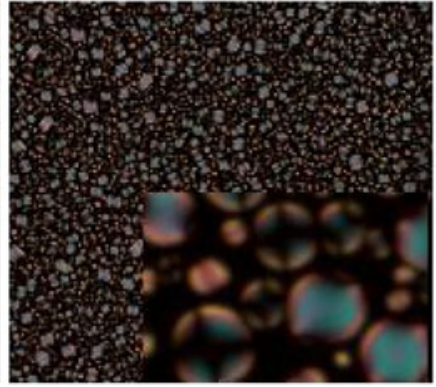

(d)

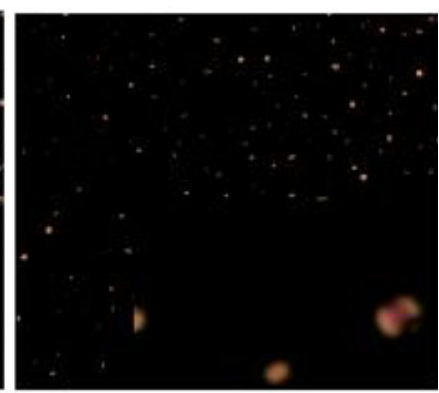

(h)

Figure 6. Droplet morphologies of DD-PDLC films having $80 \mathrm{wt} \% \mathrm{LC}$ and $0.4 \%$ dye contents on heating from: (a) $30^{\circ} \mathrm{C},(\mathbf{b}) 40^{\circ} \mathrm{C}$, (c) $62 \cdot 7^{\circ} \mathrm{C}$, (d) $67^{\circ} \mathrm{C},(\mathbf{e}) 69^{\circ} \mathrm{C}$, (f) $69 \cdot 7^{\circ} \mathrm{C},\left(\right.$ g) $70 \cdot 5^{\circ} \mathrm{C}$ and (h) $70 \cdot 7^{\circ} \mathrm{C}$.
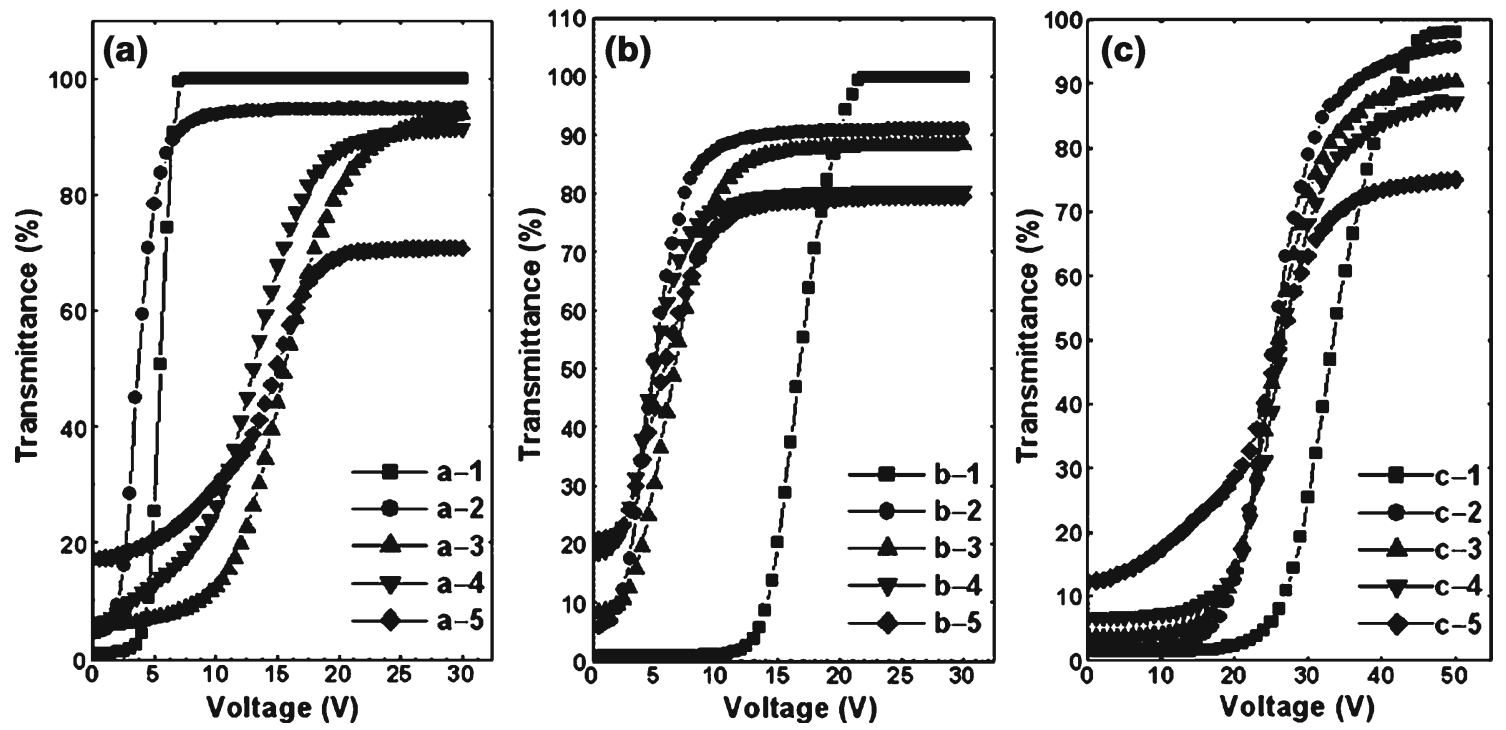

Figure 7. Applied voltages dependent light transmission of DD-PDLC films at different dyes $(0 \cdot 1$ wt $\% ; 0.2$ wt $\%$; $0.3 \mathrm{wt} \% ; 0.4 \mathrm{wt} \%$ and $1 \mathrm{wt} \%$ ) and LCs concentrations: (a) 80/20 wt $\%$, LC/monomer, (b) 78/22 wt $\%$ and (c) $76 / 22 \mathrm{wt} \%$. 
than higher dye concentration samples. Moreover the minimum transmittance was increased with the addition of dye for DD-PDLC films for all LC wt\%. In addition, the stiffness of $\mathrm{V}-\mathrm{T}$ chart was decreased with the decrease of LC contents and with the addition of dye.

Contrast ratio ( $C R=T_{0} / T_{\text {sat }}$, of a dichroic PDLC film) is an important parameter to measure the performance of any electro-optic display. Contrast ratio is a function of dye concentration and applied voltage and its values are plotted in figure 8 and shown in table 2 . The contrast ratios $(C R)$ of dichroic dye PDLC as measured at various applied voltages (figure 8) show an increase in CR with the increase of applied voltage, and attained a maximum value and further maintained constant values as well. Figure 8 shows that contrast ratio of DD-PDLC is decreased with the addition of dye for all LC wt\%. General analysis of the figure 8 shows that as compared to high dye contents, low dye contents exhibit high contrast ratio and good stiffness. It was earlier stated that high dye concentration exhibited low transmittance. Hence showed low contrast ratio due to higher absorption of UV light by dye molecules. In addition figure 8 shows an increase in contrast ratio with the decrease in LC contents.

These behaviours of decrease in $C R$ with the addition of dye and increase in contrast ratio with the decrease in LC

Table 2. A voltage-transmittance data of dye-doped PDLC films.

\begin{tabular}{|c|c|c|c|c|c|c|}
\hline Sample & Droplet size $(\mu \mathrm{m})$ & $T_{0}(\%)$ & $T_{\text {sat }}(\%)$ & $C R(30 \mathrm{~V})$ & $V_{\mathrm{th}}(\mathrm{V})$ & $V_{\text {on }}(\mathrm{V})$ \\
\hline a-1 & $2 \cdot 77$ & $1 \cdot 10$ & 100 & $90 \cdot 91$ & 4 & 8 \\
\hline$a-2$ & $6 \cdot 25$ & $4 \cdot 37$ & $94 \cdot 79$ & $21 \cdot 69$ & 2 & 10 \\
\hline$a-3$ & $9 \cdot 8$ & $5 \cdot 80$ & $93 \cdot 76$ & $16 \cdot 17$ & 11 & 24 \\
\hline$a-4$ & $25 \cdot 8$ & $6 \cdot 38$ & 91.42 & $14 \cdot 33$ & 11 & 22 \\
\hline$a-5$ & 29 & $17 \cdot 29$ & $70 \cdot 83$ & $4 \cdot 10$ & 13 & 20 \\
\hline b-1 & 1.25 & 0.85 & $99 \cdot 69$ & $117 \cdot 28$ & 14 & 22 \\
\hline$b-2$ & $9 \cdot 36$ & $5 \cdot 6$ & $90 \cdot 95$ & $16 \cdot 24$ & $2 \cdot 5$ & 12 \\
\hline$b-3$ & $10 \cdot 6$ & $8 \cdot 28$ & $88 \cdot 31$ & $10 \cdot 66$ & $3 \cdot 5$ & 14 \\
\hline b-4 & $11 \cdot 5$ & $18 \cdot 5$ & $80 \cdot 44$ & $4 \cdot 35$ & $3 \cdot 5$ & 13 \\
\hline$b-5$ & $13 \cdot 13$ & $20 \cdot 78$ & $79 \cdot 43$ & $3 \cdot 80$ & $3 \cdot 5$ & 15 \\
\hline$c-1$ & 0.63 & $1 \cdot 36$ & $98 \cdot 00$ & $18 \cdot 67$ & 24 & 45 \\
\hline$c-2$ & $0 \cdot 83$ & 1.66 & $95 \cdot 73$ & $47 \cdot 59$ & 18 & 43 \\
\hline$c-3$ & $1 \cdot 6$ & $3 \cdot 58$ & $90 \cdot 22$ & $20 \cdot 42$ & 19 & 43 \\
\hline$c-4$ & $2 \cdot 5$ & 6.77 & $87 \cdot 13$ & $10 \cdot 07$ & 20 & 44 \\
\hline$c-5$ & $5 \cdot 5$ & $12 \cdot 37$ & $75 \cdot 07$ & $5 \cdot 10$ & 21 & 38 \\
\hline
\end{tabular}
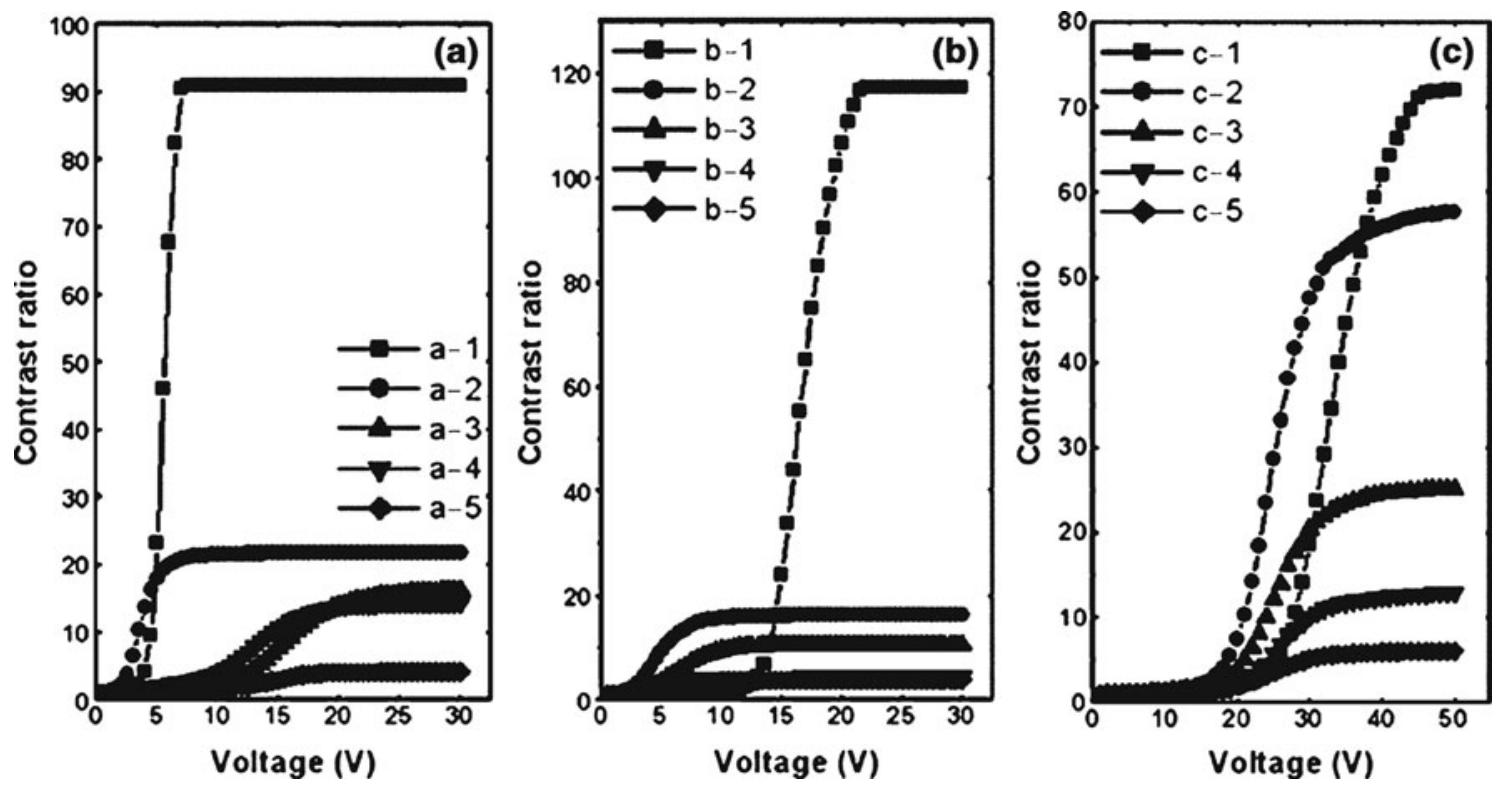

Figure 8. The dichroic dye concentration dependent contrast ratio of DD-PDLC films as a function of dye contents and applied voltages. (a) 80/20 wt \%, LC/monomer, (b) 78/22 wt \% and (c) 76/22 wt \%. 
contents of DD-PDLC are more clearly seen in figure 9. Table 2 numerically shows these results, as DD-PDLC with $76 \mathrm{wt} \% \mathrm{LC}$ contents attains high values of $C R(1: 4 \cdot 7)$ for

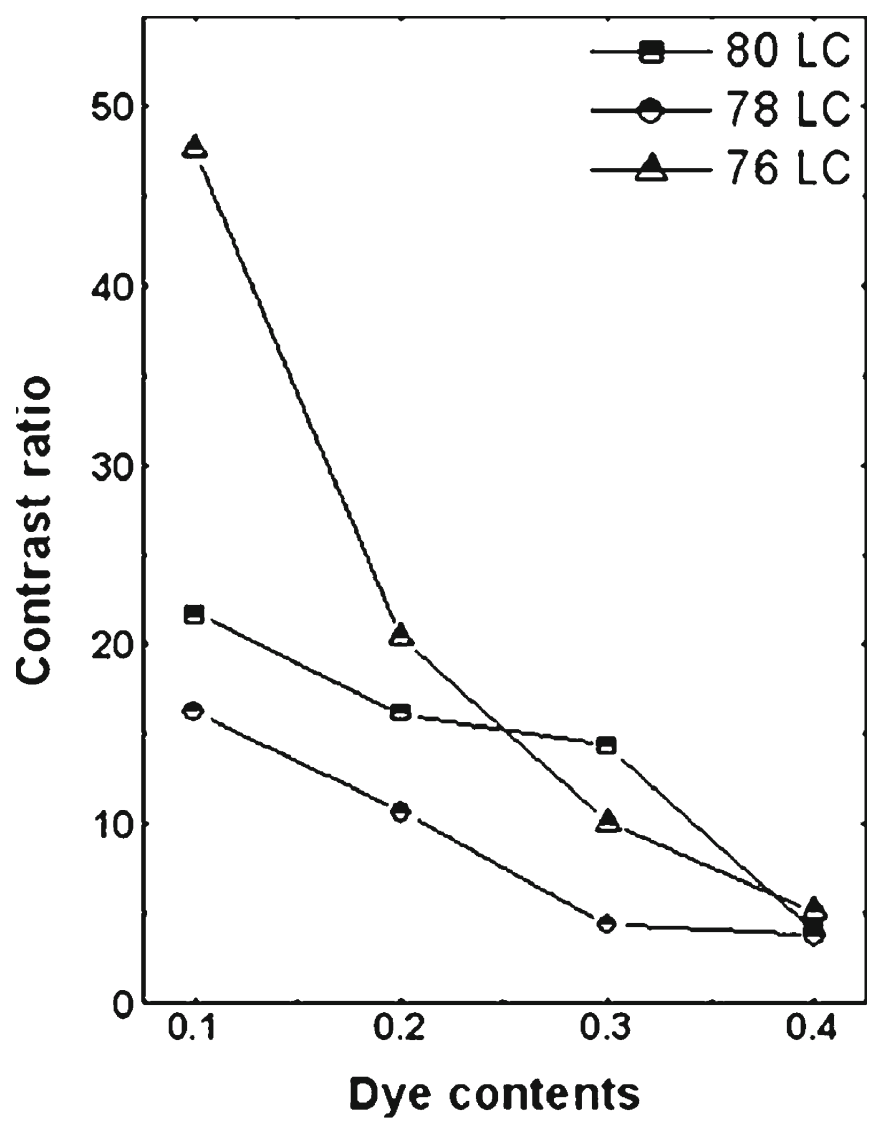

Figure 9. The contrast ratio of DD-PDLC with various dye contents. the $0 \cdot 1 \mathrm{wt} \%$ dye at worthily low threshold voltage, which is higher than the value observed by planner aligned parallel polarized dye-LC guest-host cell for the same dye contents. It was found from figure 9 and table 2 that $80 \mathrm{wt} \% \mathrm{LC}$ contents with $0 \cdot 1$ wt $\%$ dye molecules showed the $C R(1: 2)$ at 2 threshold voltage. This interesting behaviour implied the important role of LC weight fractions in enhancement of $C R$ of DD-PDLC. The lower contrast ratio observed for high LC weight fraction and higher $C R$ for low LC weight fraction could be due to the absorption of UV light by dye molecules which in turn produce poor polymerization of monomer at high LC contents. This problem was overcome by the decrease in LC weight fractions. The complete polymerization was seen by formation of well-defined LC droplet morphology in figure 2. Hence the decrease in droplet size was observed for the decrease in LC weight fraction. Further the LC droplet size was found to be independent of electric field and temperature.

The polarized optical textures of dichroic PDLC was showed predominantly bipolar configuration in comparison to other configurations (radial, axial) as shown in figure 6 . For bipolar configuration, expression for $V_{\text {th }}$ and $V_{\text {on }}$ were given by Farzana et al (2011) and Malik and Raina (2010), as

$$
\begin{aligned}
& V_{\text {th }} \propto \sqrt{\frac{K}{\Delta \varepsilon}}, \\
& V_{\text {on }}=\frac{d}{R} \sqrt{\frac{K\left(\omega^{2}-1\right)}{\varepsilon_{0} \Delta \varepsilon},}
\end{aligned}
$$

where $d$ is the film thickness, $R$ the average droplet radius, $K$ splay elastic constant, $\omega$ the aspect ratio of droplet, $\varepsilon_{0}$ the vacuum dielectric constant and $\Delta \varepsilon$ the dielectric anisotropy of the LCs. The threshold voltage $V_{\text {th }}$ depends on the elastic

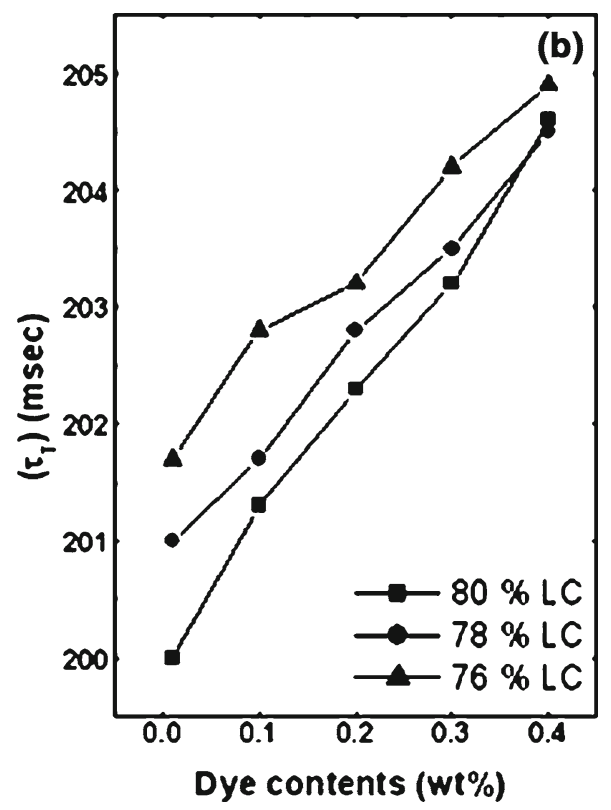

Figure 10. Response time of DD-PDLC film with $80 \mathrm{wt} \%$ LC contents: (a) against applied voltage and (b) with different dye contents. 
constant and dielectric anisotropy. Bipolar droplets exist when the splay elastic constant is smaller than the bend elastic (Yang et al 2010).

Table 2 shows the threshold voltage and $V_{\text {on }}$ values for different dye contents. It was found that addition of small amount of dye $(0.1 \mathrm{wt} \%)$ in pure PDLC leads to decrease the threshold voltage of DD-PDLC drastically for all LC weight fractions. On contrary to this behaviour, further addition of dye molecules lead to increase in threshold voltage. This is the reason that $K$, splay elastic deformation increases when dye dissolved into LC droplets. The (2) showed that the driving voltage, $V_{\text {on }}$ depends on droplet size, elastic constant and dielectric anisotropy. According to Yang et al (2010) $V_{\text {on }}$ is inversely proportional to droplet diameter, as long as the droplet size is small compared with film thickness. However, in this system for bigger the droplet size, the larger the $V_{\text {on }}$ observed. This could be due to the effect of increase in splay deformation is larger than that of droplet size increase. In addition, as the droplet size approaches the size of the cell gap, $V_{\text {on }}$ increases because the droplets shape deformation increases and droplet aspect ratio increases. However, for very small droplet size $V_{\text {on }}$ increases due to high surface anchoring energy of very small droplet size.

The optical response or response time is the sum of the total time $\left(t_{\mathrm{on}}+t_{\mathrm{off}}\right)$ for the PDLCs. The rise time $\left(t_{\mathrm{on}}\right)$ is the time required for the film to approach $90 \%$ transmittance from $10 \%$ of the off-state transmittance. The delay time $\left(t_{\text {off }}\right)$ is the time needed for film to reach $10 \%$ transmittance from $90 \%$ of the on-state transmittance. The smaller sum of these two values indicated the faster response time of the films. In general response times of PDLCs are principally governed by droplet size and shape, viscosity, elastic constants, anchoring forces and the ratio of the applied voltage over threshold voltage (Grosicka and Mucha 2000; Malik and Raina 2004). According to the theory (Kelly and Seekola 1990), response time can be described as follows:

$$
\begin{aligned}
& \frac{1}{\tau}=\frac{1}{\gamma}\left[\Delta \varepsilon E^{2}+\frac{K\left(\omega^{2}-1\right)}{R^{2}}\right], \\
& \tau_{\text {total }} \cong \frac{\gamma}{\Delta \varepsilon E^{2}},
\end{aligned}
$$

where $\tau$ is the response time, $\gamma$ the rotational viscosity coefficient, $E$ the applied electric field and the other symbols indicate the same meaning as defined earlier. The dependence of response time of pure PDLC and dye-doped PDLC on voltage and dye contents at 25 voltage is shown in figure 10 (a, b), respectively. Figure 10(a) shows that the total response time decreased with the increase of voltage for $80 \mathrm{wt} \% \mathrm{LC}$ contents for all dye contents and other all LC weight fractions follow the same behaviour as predicted by the theory (Kelly and Seekola 1990) (Eq. 4). It was also found that lower dye contents showed faster response time. The same behaviour explained well in figure 10(b). It is shown in figure 10(b) that total response time of DD-PDLC increased with the increase of dye contents and decreased with the increase of LC contents.

\section{Conclusions}

In this work, nonionic diazo dye-doped PDLC were investigated with the PIPS method. The maximum absorbance, contrast ratio, dichroic ratio and order parameter of nonionic diazo dye in nemetic host (TL203) were investigated using UV-Vis polarized spectroscopy. For this purpose a planner aligned (homogenous) cell was used. The polarized guesthost cell showed $C R$, dichroic ratio and order parameter values as $1.245,1.336$ and $0 \cdot 1$, respectively. The SBB dye in TL203 LC showed maximum absorbance at 420 and $600 \mathrm{~nm}$ wavelengths. The effect of dye contents and LC weight fractions on morphology and electro-optical properties of DDPDLC were also considered. The obtained results predict that the use of nonionic dye can significantly enhance the electro-optical properties of DD-PDLC with suitable selection of LC and dye weight fraction. Generally it was found that usage of dye helped to reduce the threshold voltage value needed for switching the material from a scattered to a transparent state. However, threshold voltage increased with the increase of dye contents which could be due to absorption of UV light by dye molecules which resulted in poor polymerization of monomers. Hence our findings illustrate that low dye $(0.1 \%)$ concentration in DD-PDLC decreased the threshold voltage, improved the transmittance, enhanced the contrast ratio and showed better electro-optical response. On the contrary high dye concentrations $(0 \cdot 3-0.4 \mathrm{wt} \%)$ tends to increase the threshold voltage and deteriorated the transmittance. Whereas the low LC weight fraction showed the higher contrast ratio due to formation of well-defined LC droplet morphology. Thus the appropriate selection of weight fraction of dye and LC could give the desired morphology and electro-optical property of DD-PDLC.

LC droplets found in these films are predominately of the bipolar configuration. However, different configurations of LC droplets, namely, spheroidal, toroidal and radial were also observed for dichroic PDLC containing $0.4 \% \mathrm{w} / \mathrm{w}$ dye. The effect of applied voltage on droplet morphology under the polarizing microscope was also observed. It was found that the bipolar droplets altered their colour and configuration to maltase-type crosses with the voltage. In addition to maltase-type crosses an intermediate, reversible and unstable twist deformation appeared as a means of reducing the bipolar structural energy. Such configurations on removing the voltage return to bipolar configuration. The phase transition temperature, $T_{\mathrm{N}-\mathrm{I}}$ with the addition of dye was observed and the images showing the effect of temperature on droplet morphology were also discussed. It was observed that the DD-PDLC films at room temperature exhibit the bipolar configuration with two point defects on the corner at high anchoring and low kinetic energy, as the temperature increased the increase in kinetic energy of LC droplets and decreased in surface anchoring energies of DD-PDLC lead to mainly spheroidal and bipolar configuration showing cross in the centre. The induced temperature also changes the colour of LC droplets. The phase transition temperature of LC first decreased with the small addition of dye and further 
it was found to be independent of dye concentration. Such behaviour remains unaltered with further addition of dye molecules, which was supposed to be due to the absorption of UV light and heat energy by dye molecules.

\section{References}

Carter S A, Le Grange J D, White W, Boo J and Wiltzius P 1997 J. Appl. Phys. 819

Denk W, Strickler J H and Webb W W 1990 Science 24873

Drzaic P S 1995 Liquid crystal dispersions (Singapore: World Scientific) Vol. 1, 22

Farzana A et al 2011 J. Appl. Polym. Sci. 1211424

Fergason J L 1984 US Patent No. 4435047

Fergason J L 1985 SID Symposium Digest 1668

Fergason J L and Calif A 1983 US Patent No. 4596445

Fuh Andy Y-G, Lee C-R and Mo T-S 2004 Mol. Cryst. Liq. Cryst. 413579

Ghanadzadeh M S and Zakerhamidi H T 2004 J. Mol. Liq. 109143

Grosicka E and Mucha M 2000 Mol. Cryst. Liq. Cryst. 353417

Hall J E and Higgins D A 2003 Polym. Mater. Sci. Eng. 88186

Han J-W, Kang T J and Park G 2000 J. Korean Phys. Soc. 36156

Kelly J and Seekola D 1990 Proc. SPIE 125717

Kumar P and Raina K K 2007 Curr. Appl. Phys. 7636

Kumar P, Raina K K and Malik P 2006 Bull. Mater. Sci. 29599

Lee S H, Lim T K, Shin S T and Park K S 2002 Jpn. J. Appl. Phys. 41208

Lin Y H, Ren H and Wu S T 2004 Appl. Phys. Lett. 844083

Liu Y J, Zheng Y B, Shi J, Huang H, Walker T R and Huang T J 2009 Opt. Lett. 342351
Lovinger J, Amundson K R and Davis D D 1994 Chem. Mater. 61726

Malik P and Raina K K 2004 Opt. Mater. 27613

Malik P and Raina K K 2010 Physica B 405161

Malik P, Raina K K and Bubnov A M 2008 Mol. Cryst. Liq. Cryst. 494242

Masutani A, Roberts T, Schüller B, Hollfelder N, Kilickiran P, Nelles G and Yasuda A 2006 Appl. Phys. Lett. 89183514

Mei E and Higgins D A 1998 Langmuir 141945

Montgomery G P and Vaz N A 1987 Appl. Opt. 26738

Montgomery Jr G P, West J L and Tamura-Lis W J $1991 \mathrm{~J}$. Appl. Phys. 691605

Petti L, Abbate G, Blau W J, Mancarella D and Mormile P 2002 Mol. Cryst. Liq. Cryst. 375785

Rochon P, Gosselin J, Natansohn A and Xie S 1992 Appl. Phys. Lett. 604

Simoni F 1997 Non linear optical properties of LC \& PDLCs (Singapore: World Scientific)

Simoni F and Francescangeli O 2000 Int. J. Polym. Mater. 45381

Springer G H and Higgins D A 2000 J. Am. Chem. Soc. 1226801

Sutherland R L, Tondiglia V P, Natarajan L V, Bunning T J and Adams W W 1994 Appl. Phys. Lett. 641074

Urbas A, Tondiglia V, Natarajan L, Sutherland R, Yu H, Li J-H and Bunning T 2004 J. Am. Chem. Soc. 12613580

Wen C-H, Gauza S and Wu S-T 2004 Liq. Cryst. 311479

West J L and Ondris-Crawford R 1991 J. Appl. Phys. 703785

Wu S T and Fuh A Y G 2005 Jpn. J. Appl. Phys. 44977

Xu W, Zipfel J B, Shear Williams R M and Webb W W 1996 Proc. Nat. Acad. Sci. 9310763

Yang K-J, Lee S-C and Choi B-D 2010 Jpn. J. Appl. Phys. 49 05EA05

Zhou J, Petti L, Mormile P and Roviello A 2003 Opt. Commun. 231263 